\title{
Efecto de Cuatro Diluciones de Plasma Seminal sobre la Tasa de Ovulación, Tamaño de Cuerpo Lúteo y Perfil de Progesterona en Alpacas
}

\author{
Effect of Four Seminal Plasma Dilutions Relating to Ovulation Rate, Corpus \\ Luteum Size and Progesterone Profile in Alpacas
}

Iván Reyna W. ${ }^{1}$, Wilfredo Huanca L. ${ }^{1,4}$, Antonio Ampuero B. ${ }^{2}$, Teodosio Huanca M. ${ }^{3}$

\section{Resumien}

\begin{abstract}
El estudio tuvo como objetivo evaluar el efecto de cuatro diluciones de plasma seminal sobre la tasa de ovulación y formación de cuerpo lúteo en alpacas. Se seleccionaron 44 alpacas sin cría al pie y con la presencia de un folículo dominante $\geq 7 \mathrm{~mm}$ detectado por ecografía transrectal. Los animales se distribuyeron al azar en cinco grupos experimentales: $\mathrm{G}_{1}$, plasma seminal (PS) diluido 1:1 (v/v) con fosfato salino bufferado (PBS); $\mathrm{G}_{2}$, PS diluido 1:2 (v/v) con PBS; $\mathrm{G}_{3}$, PS diluido 1:4 (v/v) con PBS; $\mathrm{G}_{4}$, PS diluido 1:8 (v/v) con PBS; $\mathrm{y} \mathrm{G}_{5}$, grupo control con PBS. Se aplicó $1.5 \mathrm{ml}$ de la dilución correspondiente por vía intramuscular. La tasa de ovulación y el tamaño de cuerpo lúteo fueron evaluados al segundo y noveno día, respectivamente $\left(\mathrm{D}_{0}=\right.$ inicio de tratamiento $)$ a través de ecografía transrectal. Muestras de sangre fueron tomadas los días $\mathrm{D}_{0}, \mathrm{D}_{3}, \mathrm{D}_{6}, \mathrm{D}_{9}$ y $\mathrm{D}_{12}$ para determinar niveles séricos de progesterona mediante radioinmunoanálisis. Los resultados indicaron una tasa de ovulación del $100 \%$ en los grupos tratados con diferentes diluciones de plasma seminal y $0 \%$ en el grupo control. El tamaño de cuerpo lúteo y los niveles séricos de progesterona no registraron diferencias estadísticas. Los resultados sugieren que el factor inductor de ovulación presente en el plasma seminal de alpacas tiene un efecto muy potente e induce ovulación aún en dosis bajas.
\end{abstract}

Palabras clave: alpaca, plasma seminal, diluciones, ovulación, factor inductor de ovulación

\section{Abstract}

The study was carried out to evaluate the effect of four seminal plasma dilutions on ovulation rate and corpus luteum development in alpacas. Forty four non-lactating alpacas with the presence of $\mathrm{a} \geq 7 \mathrm{~mm}$ dominant follicle detected by transrectal ultrasonography

\footnotetext{
${ }^{1}$ Laboratorio de Reproducción Animal, ${ }^{2}$ Laboratorio de Zootecnia y Producción Agropecuaria, Facultad de Medicina Veterinaria, Universidad Nacional Mayor de San Marcos, Lima, Perú

${ }^{3}$ Estación Experimental ILLPA-INIA, Puno, Perú

${ }^{4}$ E-mail: whuanca2002@yahoo.com
}

Recibido: 5 de enero de 2015

Aceptado para publicación: 18 de julio de 2015 
were selected. The animals were randomly distributed in five experimental groups: G1 $(\mathrm{n}=10)$ seminal plasma $(\mathrm{SP})$ diluted 1:1 (v/v) with phosphate buffered saline (PBS); G2 $(\mathrm{n}=10)$ SP diluted 1:2(v/v) with PBS; G3 ( $\mathrm{n}=10)$ SP diluted 1:4 (v/v) with PBS; G4 ( $\mathrm{n}=10)$ SP $1: 8(\mathrm{v} / \mathrm{v})$ with PBS; and G5 $(\mathrm{n}=4)$ control group with PBS. Adose of $1.5 \mathrm{ml}$ of the respective solution was intramuscularly administrated. Ovulation rate and corpus luteum size were evaluated at second and ninth day respectively $\left(\mathrm{D}_{0}=\right.$ day of treatment $)$ through ultrasonography. Blood samples were taken on $\mathrm{D}_{0}, \mathrm{D}_{3}, \mathrm{D}_{6}, \mathrm{D}_{9}$ and $\mathrm{D}_{12}$ to determine serum progesterone levels by radioimmunoassay. The results indicated an ovulation rate of $100 \%$ in the treated groups and $0 \%$ in the control group. The corpus luteum size and serum progesterone levels did not show statistic differences. The results indicated that the ovulation inducing factor present in the seminal plasma has a powerful effect inducing ovulation even at a low dose.

Key words: alpaca, seminal plasma, dilutions, ovulation, ovulation inducing factor

\section{INTRODUCCIÓN}

Los camélidos sudamericanos (CSA) forman parte del ambiente físico y cultural de la región altoandina del Perú, representando una importante actividad económica y social. Esta región tiene un ambiente poco flexible en términos de uso agropecuario, de allí que la alpaca y la llama son especies que brindan fibra y una fuente proteica al poblador local.

La alpaca se clasifica dentro del grupo de ovuladoras inducidas, donde el factor principal que desencadena el fenómeno de ovulación está dado por la cópula (Fernández Baca et al., 1972). También puede ser inducida por la administración de hormonas como la gonadotropina coriónica humana (hCG) (San Martín, 1968; Fernández Baca et al., 1970), hormona liberadora de gonadotropinas (GnRH) y hormona luteinizante (LH) (Bravo et al., 1992; Sumar, 1997; Leyva y García, 1999; Huanca et al., 2001).

El primer reporte sobre la existencia de un factor inductor de ovulación en camellos bactrianos fue realizado por Chen et al. (1985), quienes obtuvieron una tasa de ovu- lación del $87 \%$ al depositar artificialmente semen del camello en la vagina. Posteriormente, Pan et al. (2001) lograron aislar y purificar el factor inductor de ovulación del plasma seminal en camellos bactrianos.

Los hallazgos en camellos bactrianos condujeron a pensar que un factor inductor de ovulación podría estar presente en el plasma seminal de los CSA, debido a su relación filogenética. En el Perú, el primer reporte que sugiere la existencia de un factor inductor de ovulación fue realizado por Ríos (1989). Años después, López (2004) obtuvo una tasa ovulatoria del $100 \%$ en alpacas luego de administrar plasma seminal, vía intramuscular. Adams et al. (2005) confirmaron la existencia del factor inductor de ovulación presente en el plasma seminal de alpacas y llamas, reportando una tasa ovulatoria superior al $90 \%$, sugiriendo que el plasma seminal estimula un incremento en las concentraciones de LH.

El presente estudio fue realizado con el propósito de evaluar el efecto de cuatro diluciones de plasma seminal sobre la tasa de ovulación, tamaño de cuerpo lúteo y perfiles de progesterona en alpacas. 


\section{Materiales y Métodos}

\section{Lugar de Estudio y Animales}

El experimento se desarrolló entre los meses de enero y marzo, época de lluvias, en las instalaciones del Centro de Investigación y Producción (C.I.P) - Quimsachata, anexo de la Estación Experimental ILLPAINIA, ubicado a $4200 \mathrm{msnm}$, en el distrito de Santa Lucía, provincia de Lampa, departamento de Puno.

Se seleccionaron 44 alpacas hembras sin cría al pie, con historial reproductivo de haber tenido al menos un parto anterior y en condiciones reproductivas óptimas. El criterio de selección se basó en la presencia de un folículo dominante mayor o igual a $7 \mathrm{~mm}$ detectado por ecografía transrectal, mediante el uso de un ecógrafo modelo ALOKA SSD 500 y un transductor lineal Modo B de 7.5 MHz. Las evaluaciones ecográficas fueron realizadas por un mismo operador. Todas las hembras seleccionadas recibieron las mismas condiciones de manejo (ausencia de machos y bajo un sistema de pastoreo sobre pasturas naturales).

\section{Obtención del Plasma Seminal}

Se colectó semen de cuatro alpacas, utilizando una vagina artificial adaptada de ovino, envuelta en una frazadilla eléctrica y colocada dentro de un maniquí. Se hizo un mínimo de cinco colecciones por animal. El semen colectado fue diluido $1: 1(\mathrm{v} / \mathrm{v})$ con fosfato salino bufferado (PBS) y centrifugado a $700 \mathrm{~g}$ durante 30 minutos, para separar la fracción celular del plasma seminal. El sobrenadante, plasma seminal, se decantó y evaluó al microscopio para confirmar la ausencia de espermatozoides.

Se mezcló el plasma seminal de todos los machos para eliminar factores de variación individuales. Se le adicionó antibióticos (penicilina y estreptomicina, $10 \mu \mathrm{l} / \mathrm{ml}$ ) y se almacenó en tubos Falcon de $10 \mathrm{ml}$ bajo congelación a $-20{ }^{\circ} \mathrm{C}$.

\section{Diseño Experimental}

\section{Evaluación ecográfica pre-experimental}

La evaluación pre-experimental permitió el seguimiento de la actividad ovárica y el desarrollo de las ondas foliculares, con el propósito de seleccionar animales que presenten folículos en fase de crecimiento y con un diámetro igual o mayor de $7 \mathrm{~mm}$.

Determinación de la concentración de proteínas totales en plasma seminal

Se determinó la concentración de proteínas totales de una sub-muestra del pool de plasma seminal mediante espectrofotometría. Se utilizó un kit comercial (FAR Diagnostics, Italia) y se siguieron las indicaciones del fabricante. La medición se hizo en un analizador bioquímico semiautomático (Sinowa, China). La concentración resultante fue de 0.7 $\mathrm{g} / \mathrm{dl}$ de proteínas totales y $0.4 \mathrm{~g} / \mathrm{dl} \mathrm{de}$ albumina. Con base a estos resultados, se realizó la posterior dilución del plasma seminal con fosfato salino bufferado (PBS), según los tratamientos a ser aplicados.

\section{Tratamientos}

Tres días posteriores a la selección, se realizó una segunda ecografía a todas las hembras con la finalidad de confirmar la presencia de folículos $\geq 7 \mathrm{~mm}$. Los animales se distribuyeron aleatoriamente en cinco grupos experimentales (Cuadro 1). A los animales de cuatro grupos se les administró, vía intramuscular, $1.5 \mathrm{ml}$ del plasma seminal diluido correspondiente (1:1, 1:2, 1:4 y 1:8). Al quinto grupo se le administró PBS (grupo control).

\section{Diagnóstico ecográfico de la ovulación}

Para evaluar la tasa de ovulación en cada uno de los tratamientos se realizaron evaluaciones ecográficas el día 2 después de iniciado el tratamiento $\left(\mathrm{D}_{2}\right)$, donde se tomó como criterio de ovulación la desaparición del folículo dominante observado al inicio del tratamiento $\left(\mathrm{D}_{0}\right)$. 
Cuadro 1. Tratamientos experimentales ${ }^{1}$

\begin{tabular}{ccc}
\hline Grupo & $\begin{array}{c}\text { Tratamiento } \\
\text { (plasma seminal diluido con PBS) }\end{array}$ & Número de hembras \\
\hline G1 & $1: 1(\mathrm{v} / \mathrm{v})$ & 10 \\
G2 & $1: 2(\mathrm{v} / \mathrm{v})$ & 10 \\
G3 & $1: 4(\mathrm{v} / \mathrm{v})$ & 10 \\
G4 & $1: 8(\mathrm{v} / \mathrm{v})$ & 10 \\
G5 & PBS & 4 \\
\hline
\end{tabular}

${ }^{1}$ Se aplicó $1.5 \mathrm{ml}$ por vía intramuscular

Cuadro 2. Tamaño del cuerpo lúteo $(\mathrm{mm})$ en alpacas, al noveno día de la administración de plasma seminal diluido, vía intramuscular ( $\mathrm{n}=10$ hembras por tratamiento)

\begin{tabular}{|c|c|c|c|c|}
\hline \multirow{2}{*}{ Grupo } & \multirow{2}{*}{$\begin{array}{l}\text { Plasma seminal } \\
\text { diluido con PBS }\end{array}$} & \multirow{2}{*}{$\begin{array}{l}\text { Cuerpo lúteo } \\
(\mathrm{mm})\end{array}$} & \multicolumn{2}{|c|}{ Rango } \\
\hline & & & Inferior & Superior \\
\hline G1 & $1: 1(\mathrm{v} / \mathrm{v})$ & $12.3 \pm 2.0$ & 9 & 15 \\
\hline $\mathrm{G} 2$ & $1: 2(\mathrm{v} / \mathrm{v})$ & $12.6 \pm 1.7$ & 10 & 15 \\
\hline G3 & $1: 4(\mathrm{v} / \mathrm{v})$ & $10.7 \pm 1.7$ & 8 & 14 \\
\hline G4 & $1: 8(\mathrm{v} / \mathrm{v})$ & $10.9 \pm 1.4$ & 9 & 13 \\
\hline
\end{tabular}

Diagnóstico ecográfico del cuerpo lúteo

Una nueva evaluación ecográfica fue realizada el día 9 de iniciado el tratamiento ( $8^{\circ}$ día posovulación) con el propósito de determinar la presencia y medir el tamaño del cuerpo lúteo. La ecografía fue realizada a todas las hembras para descartar algún posible error de visualización durante la verificación de la ovulación el día $\mathrm{D}_{2}$.

Determinación de progesterona sérica por radioinmunoanálisis (RIA)

Muestras de sangre fueron tomadas a todos los animales los días $\mathrm{D}_{0}, \mathrm{D}_{3}, \mathrm{D}_{6}, \mathrm{D}_{9} \mathrm{y}$ $\mathrm{D}_{12}$. La sangre fue centrifugada y el suero resultante fue almacenado en viales de 1.5 ml y congelados hasta el análisis para determinar los niveles de progesterona mediante RIA, utilizando un kit comercial (DPC, EEUU).

\section{Análisis Estadístico}

Las diferencias en el tamaño del folículo dominante por tratamiento se analizaron mediante el análisis de varianza de una vía; las diferencias en la tasa de ovulación mediante la prueba de Fisher exacta; y las diferencias en el tamaño del cuerpo lúteo al noveno día mediante Modelos Lineales Generalizados (GLM) con medias repetidas. En el análisis estadístico se utilizó el programa SPSS v. 19. 
Cuadro 3. Concentración de progesterona sérica $(\mathrm{ng} / \mathrm{ml})$ en alpacas ovuladas por efecto de la administración intramuscular de plasma seminal diluido

\begin{tabular}{ccccccc}
\hline \multirow{2}{*}{ Grupo } & \multirow{2}{*}{$\begin{array}{c}\text { Muestras } \\
\text { (n) }\end{array}$} & $\mathrm{D}_{0}$ & $\mathrm{D}_{3}$ & $\mathrm{D}_{6}$ & $\mathrm{D}_{9}$ & $\mathrm{D}_{12}$ \\
\cline { 3 - 7 } & 10 & $0.05 \pm 0.02$ & $0.17 \pm 0.02$ & $2.52 \pm 0.19$ & $4.84 \pm 0.20$ & $0.75 \pm 0.24$ \\
$\mathrm{G} 1$ & 10 & $0.05 \pm 0.01$ & $0.14 \pm 0.02$ & $2.18 \pm 0.15$ & $4.66 \pm 0.26$ & $0.55 \pm 0.19$ \\
$\mathrm{G} 2$ & 10 & $0.05 \pm 0.01$ & $0.15 \pm 0.02$ & $1.66 \pm 0.45$ & $4.05 \pm 0.29$ & $0.29 \pm 0.11$ \\
$\mathrm{G} 3$ & 10 & $0.05 \pm 0.14$ & $0.14 \pm 0.03$ & $1.62 \pm 0.28$ & $4.19 \pm 0.19$ & $0.50 \pm 0.31$ \\
$\mathrm{G} 4$ & 4 & $0.05 \pm 0.02$ & & & $0.04 \pm 0.01$ & \\
G5 & & & & & & \\
\hline
\end{tabular}

\section{Resultados}

Las ecografías de los animales en el día $\mathrm{D}_{0}$ indicaron que todos folículos eran mayores de $7 \mathrm{~mm}$ de diámetro, observándose el menor diámetro promedio en los grupos G3 y G5 $(8.5 \mathrm{~mm})$ y el mayor promedio en el grupo G4 $(9.0 \mathrm{~mm})$, aunque sin diferencias estadísticas entre grupos.

Todos los animales de los cuatro grupos tratados con diferentes diluciones de plasma seminal llegaron a ovular en comparación con las hembras que recibieron PBS (G5) donde no hubo ninguna ovulación. El diámetro del cuerpo lúteo varió entre $10.7 \pm 1.70$ en G3 a $12.6 \pm 1.7$ en G2 (Cuadro 2), no existiendo diferencia estadística entre tratamientos.

Se obtuvieron niveles basales de progesterona sérica los días $\mathrm{D}_{0}$ y $\mathrm{D}_{3}$. La concentración de progesterona en los 4 primeros grupos comenzó a elevarse en D6 y llegó a los máximos valores en D9 (Cuadro 3), en tanto que los niveles de progesterona en el grupo G5 siempre fueron basales. No se encontró diferencia estadística entre los grupos que presentaron ovulaciones.

\section{Discusión}

El tamaño del folículo dominante tomado como criterio de selección ( $>7 \mathrm{~mm}$ ) fue considerado de acuerdo al estudio de Bravo et al. (1991), quienes indican que la ovulación no ocurre en hembras con folículos menores de $7 \mathrm{~mm}$ en el momento de la cópula.

Los resultados del presente estudio señalan que el plasma seminal diluido y aplicado vía intramuscular causa la ovulación en el $100 \%$ de las alpacas, confirmando otros reportes (López, 2004; Adams et al., 2005; Vásquez, 2005).

Los resultados sugieren que todas las diluciones del plasma seminal empleadas en el presente estudio contienen niveles adecuados del factor inductor de ovulación, lo que ocasionó que todas las alpacas ovulen y formen cuerpos lúteos con capacidad secretora de progesterona, resultados que son similares a los hallados por Tanco (2008), quien determinó que dosis de $500 \mu \mathrm{g}$ (correspondiente a $1 / 6$ de un eyaculado normal) conllevan a la formación de cuerpos lúteos con mayor diámetro en comparación con dosis de $60 \mu \mathrm{g}$ (1/50 de un eyaculado normal), indicando que la ovulación y formación del cuerpo lúteo es dependiente de la dosis del factor inductor de ovulación.

Adams et al. (2005) demostraron que el fenómeno ovulatorio es mediado por la hipófisis mediante una oleada preovulatoria de LH, y reportando un incremento notorio de LH en alpacas hembras tratadas con plas- 
ma seminal $1 \mathrm{~h}$ después del tratamiento, permaneciendo con niveles constantes durante $8 \mathrm{~h}$.

El máximo tamaño del cuerpo lúteo ocurrió el día 9 del tratamiento, sin que hubiese diferencias estadísticas entre grupos. Este resultado coincide con los resultados de desarrollo luteal presentados por Fernández Baca et al. (1970) y Adams et al. (1991). Asimismo, la mayor concentración de progesterona fue registrada al día 9 del tratamiento, correspondiente al octavo día de la ovulación. Al respecto, Sumar (1988) determinó que una concentración de progesterona mayor de $1 \mathrm{ng} / \mathrm{ml}$ a los 7 días del servicio era indicativo de ovulación y actividad luteal. La ausencia de diferencia estadística en el tamaño del cuerpo lúteo y en los niveles de progesterona entre grupos indica que las diluciones empleadas en el estudio del plasma seminal tendrían similar capacidad de inducir ovulación y formación de cuerpo lúteo, aunque Tanco (2008) pudo encontrar diferentes respuestas de progesterona sérica empleando diferentes dosis del factor inductor purificado.

El grupo G5, que recibió administración de PBS vía intramuscular, no evidenció ovulación alguna ni cambios en los niveles de progesterona sérica, coincidiendo con resultados de otros autores que aplicaron similar solución de PBS a alpacas (López, 2004; Adams et al., 2005; Vásquez, 2005; Panéz, 2007).

\section{Conclusiones}

- Diluciones crecientes de plasma seminal aplicadas intramuscularmente a alpacas hembras indujeron la ovulación y formación de cuerpo lúteo en todos los animales.

- No se encontraron diferencias estadísticas entre grupos en relación al diámetro del cuerpo lúteo o concentración de progesterona.

\section{Literatura Citada}

1. Adams GO, Sumar J, Ginther OJ. 1991. Form and function of the corpus luteum in llamas. Anim Reprod Sci 24: 127-138. doi: 10.1016/0378-4320(91) 90088-H

2. Adams GP, Ratto MH, Huanca W, Singh J. 2005. Ovulation-inducing factor in the seminal plasma of alpacas and llamas. Biol Reprod 73: 452-457. doi: 10.1095/biolreprod.105.040097

3. Bravo W, Stabenfeldt G, Lasley B, Fowler M. 1991. The effect of ovarian follicle size on pituitary and ovarian responses to copulation in domesticated South American camelids. Biol Reprod 45: 553-559. doi: 10.1095/biolreprod45.4.553

4. Bravo W, Stabenfeldt G, Fowler M, Lasley B. 1992. Pituitary response to repeated copulation and/or gonadotropinreleasing hormone administration in llamas and alpacas. Biol Reprod 47: 884888.

5. Chen B, Yuen Z, Pan G 1985. Semeninduced ovulation in the bactrian camel (Camelus bactrianus) J Reprod Fert 74: 335-339. doi: 10.1530/jrf.0.0740335

6. Fernández-Baca S, Madden D, Novoa C. 1970. Effect of different mating stimuli on induction of ovulation in the alpaca. J Reprod Fert 22: 261-267. doi: 10.1530/jrf.0.0220261

7. Fernández-Baca S, Sumar J, Novoa C. 1972. Actividad reproductiva de la alpaca mantenida en separación del macho. Memorias ALPA 7: 7-18.

8. Huanca W, Cárdenas O, Olazábal C, Ratto M, Adams G. 2001. Efecto hormonal y empadre sobre el intervalo a la ovulación en llamas. Rev Inv Pec Supl 1:462-463.

9. Leyva V, García W. 1999. Efecto de la GnRH sobre la fertilización y sobrevivencia embrionaria en alpacas. En: II Congreso Mundial sobre Camélidos. Cusco, Perú. 
10. López A. 2004. Inducción de la ovulación en llamas mediante la administración intramuscular del plasma seminal proveniente de llama, alpaca y bovino. Tesis de Médico Veterinario. Lima: Univ Nacional Mayor de San Marcos. 41 p.

11. Pan G, Chen Z, Liu X, Li D, Xie Q, Ling F, Fang L. 2001. Isolation and purification of the ovulation-inducing factor from seminal plasma in the Bactrian camel (Camelus bactrianus) Theriogenology. 55: 1863-1879.

12. Panéz S. 2007. Efecto del sitio de deposición del plasma seminal sobre la tasa de ovulación y formación del cuerpo lúteo en alpacas. Tesis de Médico Veterinario. Lima: Univ Nacional Mayor de San Marcos. 64 p.

13. Ríos M. 1989. Presencia de un factor de inducción de la ovulación en el semen de alpaca y toro. Tesis de Médico Veterinario. Lima: Univ Nacional Mayor de San Marcos. 30 p.

14. San Martín M, Copaira M, Zúñiga J, Rodríguez, R, Bustinza G, Acosta L.
1968. Aspects of reproduction in the alpaca. J Reprod Fert 16: 395-399. doi: 10.1530/jrf.0.0160395

15. Sumar J. 1988. Removal of the ovaries or ablation of the corpus luteum and its effect on the maintenance of gestation in the alpaca and llama. Acta Vet Scan 83 (Suppl): 133-141.

16. Sumar J. 1997. Avances y perspectivas en reproducción de camélidos. En: Memorias I Symposium Internacional Avances en Reproducción de Rumiantes. Lima.

17. Tanco V. 2008. Biological characterization of ovulation-inducing factor (OIF) in llama seminal plasma. Master of Science Thesis. Canada: University of Saskatchewan. $72 \mathrm{p}$.

18. Vásquez M. 2005. Efecto del plasma seminal separado en base al peso molecular, sobre la inducción de ovulación en llamas. Tesis de Médico Veterinario. Lima: Univ Nacional Mayor de San Marcos. 50 p. 